\title{
ON THE LOG DISCREPANCIES IN TORIC MORI CONTRACTIONS
}

\author{
VALERY ALEXEEV AND ALEXANDER BORISOV
}

(Communicated by Ken Ono)

\begin{abstract}
It was conjectured by $\mathrm{M}^{\mathrm{c}}$ Kernan and Shokurov that for all Mori contractions from $X$ to $Y$ of given dimensions, for any positive $\varepsilon$ there is a positive $\delta$ such that if $X$ is $\varepsilon$-log terminal, then $Y$ is $\delta$-log terminal. We prove this conjecture in the toric case and discuss the dependence of $\delta$ on $\varepsilon$, which seems mysterious.
\end{abstract}

\section{INTRODUCTION}

The main subject of this paper is the 2003 conjecture of James $\mathrm{M}^{\mathrm{c}}$ Kernan:

Conjecture 1.1 ( $\mathrm{M}^{\mathrm{c}}$ Kernan). For fixed positive integers $m, n$ and a real number $\varepsilon>0$ there exists $a \delta=\delta_{m, n}(\varepsilon)>0$ such that the following holds: Let $X$ be $a \mathbb{Q}$-factorial variety and let $f: X \rightarrow Y$ be a Mori fiber space with $\operatorname{dim} Y=n$, $\operatorname{dim} X=m+n$. Assume that $X$ is $\varepsilon$-log terminal. Then $Y$ is $\delta$-log terminal.

A related stronger conjecture was suggested by V.V. Shokurov. Let $f: X \rightarrow Y$ be a proper surjective morphism with connected fibers of normal varieties so that $X / Y$ is of relative Fano type (see definitions below) and let $\Delta$ be a $\mathbb{Q}$-divisor on $X$ such that $K_{X}+\Delta=f^{*} L$ for some $\mathbb{Q}$-divisor $L$ on $Y$.

By Kawamata's subadjunction formula [Kaw97, Kaw98, see also [Amb05], one has $K_{X}+\Delta=f^{*}\left(K_{Y}+R+B\right)$, where $R$ is the discriminant part and $B$ is the "moduli" part, a $\mathbb{Q}$-divisor defined only up to $\mathbb{Q}$-linear equivalence.

Conjecture 1.2 (Shokurov). In the above settings, assume that $(X, \Delta)$ is $\varepsilon$-log terminal. Then there exists $\delta=\delta_{m, n}(\varepsilon)>0$ and an effective moduli part $B$ such that $(Y, R+B)$ is $\delta$-log terminal.

Conjecture 1.2 clearly implies Conjecture 1.1, for a Mori fiber space consider a large integer $N \gg 0$ and a generic element $D$ of a very ample linear system $-N K_{X}+f^{*} M$ for some $M$ on $Y$, and let $\Delta=\frac{1}{N} D$. Then $K_{X}+\Delta=f^{*} L$ and for the minimal log discrepancies one has

$$
\operatorname{mld}(X, \Delta)=\operatorname{mld}(X)+\frac{1}{N} \quad \text { and } \quad \operatorname{mld}(Y, R+B) \leq \operatorname{mld}(Y) .
$$

Taking the limit $N \rightarrow \infty$ gives the implication.

We refer the reader to KM98 for basic definitions and results of the Minimal Model Program, some of which we briefly recall below. For any normal variety $X$

Received by the editors November 16, 2012.

2010 Mathematics Subject Classification. Primary 14E30, 14M25.

(c) 2014 American Mathematical Society Reverts to public domain 28 years from publication 
for which some positive multiple of the canonical class $K_{X}$ is Cartier, one defines discrepancies $a_{i} \in \mathbb{Q}$ by the formula

$$
K_{X^{\prime}}=\pi^{*} K_{X}+\sum a_{i} E_{i}, \quad \operatorname{Exc}(\pi)=\bigcup E_{i},
$$

in which $\pi: X^{\prime} \rightarrow X$ is a resolution of singularities and $E_{i}$ are the irreducible exceptional divisors of $\pi$. The $\log$ discrepancies are the numbers $a_{i}^{\log }=a_{i}+1$. The minimal log discrepancy $\operatorname{mld}(X)$ is the infinum of log discrepancies, going over all resolutions of singularities. Then either $\operatorname{mld}(X) \geq 0$ or $\operatorname{mld}(X)=-\infty$. In the first case, variety $X$ is $\log$ canonical, and $\operatorname{mld}(X)$ can be computed on any one resolution $\pi: X^{\prime} \rightarrow X$ such that $\operatorname{Exc}(\pi)$ is a normal crossing divisor.

A variety is said to be $\varepsilon$-log terminal (abbreviated below to $\varepsilon$-lt) if its log discrepancies are $>\varepsilon$, i.e. if for ordinary discrepancies one has $a_{i}>-1+\varepsilon$. Similarly, a variety is $\varepsilon$-log canonical if the $\log$ discrepancies are $\geq \varepsilon$. In particular, 0-log terminal is the same as Kawamata log terminal (klt), and 0-log canonical is the same as log canonical.

We recall that $f: X \rightarrow Y$ is a Mori fiber space if $f$ is projective, $-K_{X}$ is $f$ ample, and the relative Picard number is $\rho(X / Y)=1$. The assumption that $X$ is $\mathbb{Q}$-factorial implies that so is $Y$ (cf. KMM87, Lemma 5-1-5]).

Finally, a variety $X$ is called a variety of Fano type (FT) if there exists an effective $\mathbb{Q}$-divisor $D$ such that the pair $(X, D)$ is klt and $-\left(K_{X}+D\right)$ is nef and big.

There are numerous motivations for the above conjectures. The case $\varepsilon=\delta=0$ of Conjecture 1.1 i.e. " $X$ is klt implies $Y$ is klt", follows easily by cutting $X$ with $m$ general hyperplanes and reducing to a finite surjective morphism. Even if $X$ is not $\mathbb{Q}$-factorial, the implication " $X$ is klt implies $(Y, \Delta)$ is klt for an appropriate divisor $\Delta "$ is true, as proved by Fujino Fuj99.

The first nontrivial case with $\varepsilon>0$ appears when $\operatorname{dim} X=3$ and $\operatorname{dim} Y=2$, i.e. when $f: X \rightarrow Y$ is a singular conic bundle. Mori and Prokhorov MP08 considered the case when $X$ is terminal. In this case, they proved the Iskovskikh conjecture which says that $Y$ must have at worst Du Val singularities. This proves that one can take $\delta_{1,2}(1)=1-c$ for any $c>0$. Yuri Prokhorov also showed us several examples of conic bundles of the form $\left(\mathbb{P}^{1} \times \mathbb{A}^{2}\right) / G \rightarrow \mathbb{A}^{2} / G$ for a cyclic group $G$ which indicate that Conjecture 1.1 is plausible.

Conjecture 1.1 may also be viewed as the local analogue of the BorisovAlexeev-Borisov (BAB) boundedness conjecture [BB92, Ale94], which says that for fixed $n$ and $\varepsilon>0$ the family of $n$-dimensional $\varepsilon$-lt Fano varieties is bounded.

Indeed, if $X$ happens to be a Fano variety, then the family of possible $\varepsilon$-lt varieties $X$ is bounded by the BAB conjecture. Then the family of possible varieties $Y$ must be bounded, so some $\delta(\varepsilon)>0$ must exist. Vice versa, when trying to prove the $\mathrm{BAB}$ conjecture by induction, Conjecture 1.1 naturally appears as one of the steps. In this sense, it can be considered to be "the local BAB conjecture".

The main result of the present paper is the following:

Theorem 1.3. Conjecture 1.1 holds in the toric case, i.e. when $f: X \rightarrow Y$ is a morphism of toric varieties corresponding to a map of fans $\left(N_{X}, \Sigma_{X}\right) \rightarrow\left(N_{Y}, \Sigma_{Y}\right)$.

Note that in the toric case, if one denotes by $\Delta$ the sum of torus invariant divisors with coefficients 1 , then one has $K_{X}+\Delta=0$ and the pair $(X, \Delta)$ is log canonical with $\operatorname{mld}(X, \Delta)=0$. Thus, the more general Conjecture 1.2 does not fit the toric case very well. 
A very interesting question is to find the asymptotic of the function $\delta(\varepsilon)$ as $\varepsilon \rightarrow 0$. Concerning this, we prove the following:

Theorem 1.4. In the conditions of Theorem 1.3, suppose additionally that the generic fiber of $f$ is a finite, unramified in codimension one, toric quotient of a fixed toric Fano variety $P$. Then there exists a constant $C$ such that $\delta \geq C \cdot \varepsilon^{m+1}$.

On the other hand, we prove:

Theorem 1.5. There exists a sequence of toric Mori fiber spaces with $m=n=2$ such that $\operatorname{mld}(X) \rightarrow 0$ and $\operatorname{mld}(Y) \approx C \cdot \operatorname{mld}(X)^{4}$.

\section{Proofs of THE MAIN RESUlts}

We continue with the notation of the Introduction. We first need to examine the combinatorics of the fans of $X$ and $Y$. We refer to Ful93 or Oda88 for the general theory of toric varieties. We work over $\mathbb{C}$ for simplicity, although, as usual in toric geometry, the results remain true over a field of positive characteristic as well.

Recall that a toric variety $X$ is given by a pair $\left(N_{X}, \Sigma_{X}\right)$, where $N_{X}$ is a lattice (called the lattice of valuations) and $\Sigma_{X}$ is a rational polyhedral fan in $N_{X} \otimes \mathbb{R}$. A toric map from a toric variety $X$ to a toric variety $Y$ is given by a linear map $F: N_{X} \rightarrow N_{Y}$ such that its extension $F_{\mathbb{R}}: N_{X} \otimes \mathbb{R} \rightarrow N_{Y} \otimes \mathbb{R}$ sends every cone in the fan $\Sigma_{X}$ to a cone in the fan $\Sigma_{Y}$.

We denote by $N_{Z}$ the lattice $\operatorname{Ker}(F)$ and by $\Sigma_{Z}$ the restriction of $\Sigma_{X}$ to $\operatorname{Ker}\left(F_{\mathbb{R}}\right)$. We recall the following basic facts:

Fact 1. The morphism $f: X \rightarrow Y$ is proper iff $F_{\mathbb{R}}^{-1}\left(\operatorname{Supp} \Sigma_{Y}\right)=\operatorname{Supp} \Sigma_{X}$.

Fact 2. A general fiber of $f: X \rightarrow Y$ is a product of a toric variety of dimension $\operatorname{dim} N_{Z}$ with the finite part, the product of finitely many copies of the group schemes $\mu_{r_{i}}=\operatorname{Spec} k[z] /\left(z^{r_{i}}-1\right)$. The character group of the finite part is the torsion subgroup of $\operatorname{coker}\left(F: N_{X} \rightarrow N_{Y}\right)$.

A Mori fiber space $f: X \rightarrow Y$ is a surjective proper morphism with connected fibers, and a general fiber is connected and reduced. Therefore, in our situation one has $F_{\mathbb{R}}^{-1}\left(\operatorname{Supp} \Sigma_{Y}\right)=\operatorname{Supp} \Sigma_{X}$, and the morphism of lattices $F: N_{X} \rightarrow N_{Y}$ is surjective.

Fact 3. A toric variety $X$ is $\mathbb{Q}$-Gorenstein; i.e. the canonical divisor $K_{X}$ is $\mathbb{Q}$ Cartier iff there exists a function $\ell=\ell_{-K_{X}}: \operatorname{Supp} \Sigma_{X} \rightarrow \mathbb{R}$ which is linear on each cone $\sigma \in \Sigma_{X}$ and such that $\ell\left(P_{i}\right)=1$ for each shortest integral generator $P_{i}$ of each ray $R_{i}$ of $\sigma$.

Fact 4. A toric variety $X$ is $\mathbb{Q}$-factorial; i.e. every Weil divisor is $\mathbb{Q}$-Cartier iff the fan $\Sigma$ is simplicial, i.e. every cone is a simplex.

Fact 5. The $\operatorname{mld}(X)$ is computed as the minimum of the piece-wise linear function $\ell=\ell_{-K_{X}}$ on $\operatorname{Supp} \Sigma_{X} \cap N_{X} \backslash\{0\}$.

Obviously, our problem is local on $Y$, so we can assume that $Y$ is affine. Since $X$ and $Y$ are $\mathbb{Q}$-factorial, $Y$ is a quotient of $\mathbb{A}^{n}$ by a finite abelian group. Combinatorially, it is obtained from the standard cone $C=\left\{\left(x_{1}, \ldots, x_{n} \mid \forall x_{i} \geq 0\right\}\right.$ in $\mathbb{R}^{n}$, with the lattice being a finite extension of the standard lattice $\mathbb{Z}^{n}$. Thus, the fan $\Sigma_{Y}$ consists of the cone $C$ and its faces. 
If the shortest integral generators of the cone $C$ are the standard basis vectors $e_{i}$, then the linear function computing $\operatorname{mld}(Y)$ is simply $\ell_{-K_{Y}}=\sum x_{i}$.

Proposition 2.1. Suppose that $f: X \rightarrow Y$ is a toric Mori fiber space, with $\mathbb{Q}$-factorial $X$ and affine $Y$ as above. Denote by $F: N_{X} \rightarrow N_{Y}$ the map of the corresponding lattices, and extend it to the linear map $F_{\mathbb{R}}$ from $N_{X} \otimes \mathbb{R}=\mathbb{R}^{n+m}$ to $N_{Y} \otimes \mathbb{R}=\mathbb{R}^{n}$. We choose the basis of $N_{X} \otimes \mathbb{R}$ so that the map $F_{\mathbb{R}}$ is the projection of $\mathbb{R}^{n+m}$ to the last $n$ coordinates. Then the following is true about the fan of $X$ in $N_{X} \otimes \mathbb{R}$.

1) It has exactly $(n+m+1)$ one-dimensional cones (rays) $R_{i}, i=0,1, \ldots, m+n$, of which $R_{0}, \ldots, R_{m}$ are in $\operatorname{Ker}\left(F_{\mathbb{R}}\right)$, and for all $i=m+1, \ldots, m+n$ the ray $R_{i}$ is mapped by $F_{\mathbb{R}}$ to the ray of $C$, which is the span of the standard basis vector $e_{i-m}$.

2) Any generators of $R_{i}$ for $i=0, \ldots, m$ form a simplex that contains 0 .

3) The cones in the fan of $X$ are precisely the simplicial cones generated by $R_{i}$ for $i \in S \subset\{0,1, \ldots, n+m\}$, where $S$ does not contain $\{0,1, \ldots, m\}$.

Proof. The condition $F_{\mathbb{R}}^{-1}(C)=\operatorname{Supp} \Sigma_{X}$ implies that

(1) The fan $\Sigma_{Z}$ is complete, i.e. $\operatorname{Supp} \Sigma_{Z}=N_{Z} \otimes \mathbb{R}$. In particular, $\Sigma_{X}$ has at least $m+1$ rays in $\operatorname{ker} F_{\mathbb{R}}$.

(2) For each of the $n$ rays of $C$, there exists at least one ray of $\Sigma_{X}$ lying over it.

Recall that the Picard group of $\mathbb{R}$-Cartier divisors on a toric variety $X$ is the quotient of the space of piece-wise linear functions modulo the space of linear functions on $\Sigma_{X}$. Since both fans are simplicial (because $X, Y$ are both $\mathbb{Q}$-factorial) and full-dimensional, the relative Picard number $\rho(X / Y)$ is the difference between the number of rays of $X$ and $Y$ minus the relative dimension, $m$.

Therefore, $\Sigma_{X}$ has $m+n+1$ rays. Thus, $\Sigma_{X}$ has no other rays other than the $(m+1)+n$ rays listed above, and over each ray of $C$ there exists a unique ray of $\Sigma_{X}$. This proves 1$)$.

Finally, for this set of $n+m+1$ rays there is only one simplicial fan with support $F_{\mathbb{R}}^{-1}(C)$ : the one described in 3 ). This proves 2) and 3).

We now choose a basis in $N_{X} \otimes \mathbb{R}$ so that the last $n$ coordinate vectors are the primitive elements of $N_{X}$ on the rays $R_{i}, i \geq m+1$, denoted by $P_{i}$. For $i=0, \ldots, m$ we also denote by $P_{i}$ the primitive elements of $N_{Z}=\operatorname{ker}\left(N_{X} \rightarrow N_{Y}\right)$ on the rays $R_{i}$.

By the above proposition, the fan $\Sigma_{X}$ is isomorphic to the Cartesian product $\Sigma_{Y} \times \Sigma_{Z}$. Since a general fiber of $X \rightarrow Y$ is connected, the map $F: N_{X} \rightarrow N_{Y}$ is surjective. Therefore, one has $N_{X} \simeq N_{Y} \times N_{Z}$. However, one need not have $\left(N_{X}, \Sigma_{X}\right) \simeq\left(N_{Y}, \Sigma_{Y}\right) \times\left(N_{Z}, \Sigma_{Z}\right)$. In particular, it is possible that $F\left(P_{i}\right)$ are not primitive in the lattice $N_{Y}$.

Denote by $\Delta$ the simplex with vertices $P_{i}$ in $\operatorname{Ker}(F)$. This structure defines the toric Fano variety $Z$ of Picard number one, which is the generic fiber of $f$. We choose the coordinates in $N_{X} \otimes \mathbb{R}=\mathbb{R}^{n+m}$ so that the lattice generated by $P_{i}$ is the standard $\mathbb{Z}^{m} \subset \mathbb{R}^{m}=\operatorname{Ker}\left(F_{\mathbb{R}}\right)$; the lattice $N_{Z}$ is a finite extension of it.

We now describe our basic strategy for the proof of Theorem 1.3. Recall that $\operatorname{mld}(Y)$ of a toric variety $Y$ is computed as the minimum of the linear function $\sum_{i=1}^{n} x_{i}$ over the nonzero points of $N_{Y} \cap C$.

According to Fact [5, the mld of a toric singularity is the minimum of the log discrepancies of the nonzero points of the corresponding cone, where the log discrepancy of a point is the value on it of the linear function that equals 1 on the 
rays of the cone. Suppose that for $Y$ this minimum is achieved at some point $A$. We want to prove that if the $\log$ discrepancy of $A$ is very small, there must exist a point in $N_{X}$, in one of the cones of $\Sigma_{X}$, for which the log discrepancy is also small (less than the given $\varepsilon$ ). To look for this point, we take a preimage $P$ of $A$ in $N_{X}$ (it is possible because $N_{X} \rightarrow N_{Y}$ is surjective) and consider its multiples $P, 2 P, \ldots, t P$ modulo the lattice $\mathbb{Z}^{n+m}$, for some $t$ to be specified later. If the log discrepancy of $A$ is really small, then we can choose a fairly large $t$ such that for all these points the sum of the last $n$ coordinates is still small. By the Dirichlet Box Principle, we can choose two of these points to be close to each other, and take their difference. If we subtract in the correct order, this produces a point $Q$ in $N_{X}$ with the last $n$ coordinates nonnegative and with small sum, and the projection to the first $m$ coordinates being near the origin. Because the union of the cones for $Z$ is the whole $\mathbb{R}^{m}$, this projection must belong to some cone, which implies that $Q$ lies in some cone for $X$ and has a small log discrepancy there.

To illustrate the method, we first establish Theorem 1.3 in the particular case when $Z$ is an unramified in codimension one quotient of the usual projective space $\mathbb{P}^{m}$. This means that the barycentric coordinates of 0 in the simplex $\Delta$ are $\left(\frac{1}{m+1}, \ldots, \frac{1}{m+1}\right)$.

Proposition 2.2. In the above notation, suppose additionally that the points $P_{i}$ for $1 \leq i \leq m$ are the standard $e_{i} \in \mathbb{R}^{n+m}$, and $P_{0}=(-1, \ldots,-1 ; 0, \ldots, 0)$ (here the semicolon separates the first $m$ coordinates from the last $n)$. Then for any $\varepsilon>0$, if $\operatorname{mld}(X)>\varepsilon$, then $\operatorname{mld}(Y)>\delta=\left(\frac{\varepsilon}{2 m}\right)^{m+1}$.

Proof. Suppose that $\operatorname{mld}(Y) \leq \delta$. Denote the point in $N_{Y}$ on which the mld is achieved, by $A$. In other words, $A=\left(a_{1}, \ldots, a_{n}\right)$, where $a_{i}$ are nonnegative, not all zero, and $\sum a_{i} \leq \delta$. Because $F$ is surjective, $A=F(P)$ for some $P \in N_{X}$. Suppose $P=\left(b_{1}, \ldots, b_{m} ; a_{1}, \ldots, a_{n}\right)$. We may additionally assume that all $b_{i}$ are in $[0,1)$, because $\mathbb{Z}^{m} \subseteq N_{Z}$.

Choose $t=\delta^{-\frac{m}{m+1}}$. For all integers $k \in[0, t)$ consider the points $P_{k}=k P$ $\bmod \mathbb{Z}^{n+m}=\left(\left\lfloor k b_{1}\right\rfloor, \ldots,\left\lfloor k b_{m}\right\rfloor ; k a_{1}, \ldots, k a_{n}\right)$ and their projections to $\mathbb{R}^{m}: \bar{P}_{k}=$ $\left(\left\lfloor k b_{1}\right\rfloor, \ldots,\left\lfloor k b_{m}\right\rfloor\right)$.

Lemma 2.3. Suppose for all integers $k \in[0, t), Q_{k}=\left(b_{1, k}, \ldots, b_{m, k}\right)$ are arbitrary points in $[0,1)^{m}$. Then there exist $i$ and $j$ so that for all $l=1, \ldots, m$ we have $\left|b_{l, i}-b_{l, j}\right| \leq t^{-1 / m}$.

Proof. Identify $[0,1)^{m}$ with the quotient $\mathbb{R}^{m} / \mathbb{Z}^{m}$, with the usual Haar probability measure. For each $Q_{i}$ consider a closed box neighborhood of it defined by the conditions $x_{l} \in\left[b_{l, i}-\frac{1}{2} t^{-1 / m}, b_{i}+\frac{1}{2} t^{-1 / m}\right] \bmod Z$. The volume (i.e. the Haar measure) of each such box is $t^{-1}$. Note that the total number of points is $\lfloor t\rfloor>t$, so the total sum of the volumes is greater than 1 . Thus there exist $i$ and $j$ such that the corresponding boxes intersect. The triangle inequality in $\mathbb{R} / \mathbb{Z}$ implies the result.

We apply the above lemma to the points $Q_{k}=\bar{P}_{k}$. Without loss of generality, we can assume that $i<j$. Consider the point $Q=P_{j}-P_{i} \in N_{X}$. In coordinates, $Q=\left(\left\lfloor j b_{1}\right\rfloor-\left\lfloor i b_{1}\right\rfloor, \ldots,\left\lfloor j b_{m}\right\rfloor-\left\lfloor i b_{m}\right\rfloor ;(j-i) a_{1}, \ldots,(j-i) a_{n}\right)$. Note that $0<j-i \leq t$, so the sum of the last $n$ coordinates of $Q$ is at most $t \delta$. Suppose that for $l \geq m+1$ we have $F\left(P_{l}\right)=c_{l} \cdot e_{l-m}$. Then the contribution to the $\log$ discrepancy of $Q$ from 
the last $n$ coordinates is

$$
k \sum_{l=m+1}^{n+m} \frac{a_{l-m}}{c_{l}} \leq k\left(a_{l}+\ldots+a_{n}\right) \leq k \cdot \delta .
$$

The first $m$ coordinates of $Q$ are less than $t^{-1 / m}$ in absolute value. Denote by $\bar{Q}$ the natural projection of $Q$ to $N_{Z} \otimes \mathbb{R}$ :

$$
\bar{Q}=\left(\left\lfloor j b_{1}\right\rfloor-\left\lfloor i b_{1}\right\rfloor, \ldots,\left\lfloor j b_{m}\right\rfloor-\left\lfloor i b_{m}\right\rfloor\right)=\left(q_{1}, \ldots, q_{m}\right) .
$$

Then $\bar{Q}$ belongs to one of the cones of the fan for $Z$ as follows.

Case 1. All $q_{l}$ are nonnegative. Then $\bar{Q}$ belongs to the cone $x_{i} \geq 0$, which is the span of $P_{i}$, for $1 \leq i \leq m$. The contribution to the log discrepancy from the first $m$ coordinates is at most $m \cdot t^{-1 / m}$.

Case 2. At least one of the numbers $q_{l}$ is negative. Without loss of generality, we can assume that $q_{1}$ is the smallest (i.e. the most negative) of $q_{l}$. Then $\bar{Q}$ lies in the span of $P_{0} ; P_{2}, \ldots, P_{m}$. Its coordinates in that basis are $\left(-q_{1} ; q_{2}-q_{1}, \ldots, q_{m}-q_{1}\right)$. The contribution to the log discrepancy from the first $m$ coordinates is at most $(2 m-1) \cdot t^{-1 / m}$.

Putting it together, the $\log$ discrepancy of $Q$ is at most $(2 m-1) t^{-1 / m}+t \delta$. Since we chose $t=\delta^{-\frac{m}{m+1}}$, we get the $\log$ discrepancy of $Q$ to be at most $2 m \delta^{\frac{1}{m+1}} \leq \varepsilon$, which contradicts $\operatorname{mld}(X)>\varepsilon$.

This completes the proof of Proposition 2.2.

Remark 1. One can improve the above estimate slightly by choosing $t$ to be a suitable constant times $\delta^{-\frac{1}{m+1}}$, and by a more "projectively symmetric" estimate for $\bar{Q}$. But it will still give the result of the form $\delta \geq \operatorname{const}(m) \cdot \varepsilon^{m+1}$ and would make the exposition considerably more muddled.

Proof of Theorems 1.3 and 1.4. A slight generalization of the above argument yields Theorem 1.4. Indeed, suppose $Z$ is an arbitrary toric Fano variety of dimension $m$ with the corresponding simplex $\Delta$, and suppose that the barycentric coordinates of 0 in $\Delta$ are $y_{1}, y_{2}, \ldots, y_{m+1}$. We can fix the vertices $P_{i}, i \leq m+1$, in $\mathbb{Z}^{m}$. As before, we can take $t$ to be $\delta^{-\frac{1}{m+1}}$. We apply the same lemma (though one can get a somewhat better estimate by generalizing scaling the boxes, keeping the same volume). As a result, the absolute values of all coordinates of the point $\bar{Q}$ are again at most $t^{-\frac{1}{m}}=\delta^{\frac{1}{m+1}}$. So for each of the $(m+1)$ linear functions corresponding to the $m$-dimensional cones of the fan for $Z$, the $\log$ discrepancy for $\bar{Q}$ will be bounded by a constant multiple of $\delta^{\frac{1}{m+1}}$. The same estimate as above proves that for the fixed $y_{1}, y_{2}, \ldots, y_{m+1}$ one can choose $\delta=$ const $\cdot \varepsilon^{m+1}$, thus proving Theorem 1.4.

Finally, Theorem 1.3 follows from Theorem 1.4 by a simple observation that if $\operatorname{mld}(X)>\varepsilon$, then also $\operatorname{mld}(Z)>\varepsilon$. By the main result of BB92 (the BAB Conjecture for toric varieties) there are only finitely many possible Fano varieties $Z$ with $\operatorname{mld}(Z)>\varepsilon$.

While the above argument may seem to imply the existence of a general estimate for the $\operatorname{mld}(Y)$ in terms of $\operatorname{mld}(X)$ in the form const $(m) \cdot \varepsilon^{m+1}$, the constant depends implicitly on $\varepsilon$. In fact, one simply cannot hope for the estimate above, in light of the following example which proves Theorem 1.5. 
Example 2.4. We fix $n=m=2$. Suppose $l$ is a natural number. Consider a triangle in $\mathbb{Z}^{2}$ with vertices $(1,0),(-(l-1), 1),(-(l-1),-1)$. This gives a weighted projective space; we multiply it by $\mathbb{A}^{2}$ and consider the quotient by the group $\mu_{r}$, where $r=l^{4}+1$, given by the weights $\frac{1}{r}\left(l, l^{2} ; 1,1\right)$. In other words, we take a lattice $\mathbb{Z}^{2} \subset \mathbb{Z}^{4}$ and enlarge the latter by adjoining the point $\frac{1}{r}\left(l, l^{2} ; 1,1\right)$. The rays are $(1,0,0,0),(-(l-1), 1,0,0),(-(l-1),-1,0,0) ;(0,0,1,0),(0,0,0,1)$. The map $F$ is just the projection to the last two coordinates. The variety $Y$ is a cyclic quotient singularity of type $\frac{1}{r}(1,1)$.

We claim that for the above example the $\operatorname{mld}(Y)$ is asymptotically $\frac{1}{l^{4}}$, while $\operatorname{mld}(X)$ is asymptotically at least $\frac{1}{2 l}$. This would obviously imply Theorem 1.5.

The first part is easy: $\operatorname{mld}(Y)=2 / r$, which is asymptotically $2 / l^{4}$.

For the estimate on $\operatorname{mld}(X)$, consider the point $N=\frac{1}{r}\left(l, l^{2} ; 1,1\right)$ in $\mathbb{R}^{4}$. We need to prove that no sums $k N$ and points of $Z^{4}$ have small log discrepancy, in any of the cones of $X$. Consider such a point $Q=k P+B$, where $k$ is an integer from 1 to $r-1$ and $B \in \mathbb{Z}^{4}$. Clearly, we can assume that the last two coordinates of $B$ are zero, thus $B \in \mathbb{Z}^{2} \subset \mathbb{Z}^{4}$. Note the following:

1) If $k>\left(l^{3}\right) / 2$, then the contribution from the last two coordinates is already too big. So we are only concerned with $k \leq\left(l^{3}\right) / 2$.

2) Since $k \leq\left(l^{3}\right) / 2$, the first coordinate in $k N$ is between 0 and $1 / 2$. Therefore the points in the left cone are of no concern: they would have a log discrepancy contribution from the first two coordinates of at least $(1 / 2) /(l-1)$. For the points in the upper or lower cone, if $k>l^{2} / 2$, then the log discrepancy is at least $k l / r>$ $\left(l^{3}\right) /(2 r)$, which is about $1 /(2 l)$. So we only need to consider $k \leq l^{2} / 2$.

3) Since $k \leq l^{2} / 2$, the second coordinate of $k N$ is between 0 and about $1 / 2$. This rules out points in the lower cone. For the upper cone, we clearly need to be concerned only with the points $k N+(0,0)$. There the smallest $\left(x_{1}+l x_{2}\right)$ value is at least $1 \times l^{2} / r=l^{3} / r$, which is about $1 / l$.

\section{Miscellaneous Remarks}

It may seem like one cannot avoid using the BAB conjecture to prove Theorem 1.3. However, there is an explicit version of the toric BAB theorem (proved by Lagarias and Ziegler [LZ91, and originally by Hensley [Hen83], before [BB92]) which may probably be used to get an explicit bound of the form $\delta=C(m) \varepsilon^{d(m)}$. However, this is by no means automatic, and the correct power $d(m)$ is highly mysterious. Probably, for $m=2$ it is 4 , but in higher dimensions the answer is not obvious.

By a more careful generalization of the argument for $\mathbb{P}^{m}$, one can get an estimate for $\delta$ in terms of $m, \varepsilon$ and the Tian's alpha invariant of $Z$ (that essentially measures "asymmetry" of the simplex $\Delta$ ). Perhaps a generalization of this argument to the nontoric case will naturally use this invariant as well.

We also note a subsequent preprint Bir12 to our paper whose stated result is a proof of Shokurov's conjecture under the assumption that the pair $\left(F, \operatorname{Supp} \Delta_{F}\right)$ belongs to a bounded family, where $F$ is a general fiber and $K_{F}+\Delta_{F}=\left.\left(K_{X}+\Delta\right)\right|_{F}$, but without the toric assumption. 


\section{ACKNOWLEDGEMENTS}

This research originated in a discussion at the ACC workshop at the American Institute of Mathematics in May of 2012 as an attempt to answer a question brought up by Yuri Prokhorov. The authors wish to thank Yu. Prokhorov, V.V. Shokurov and other participants of this workshop for many fruitful discussions.

\section{REFERENCES}

[Ale94] Valery Alexeev, Boundedness and $K^{2}$ for log surfaces, Internat. J. Math. 5 (1994), no. 6, 779-810, DOI 10.1142/S0129167X94000395. MR1298994 (95k:14048)

[Amb05] Florin Ambro, The moduli b-divisor of an lc-trivial fibration, Compos. Math. 141 (2005), no. 2, 385-403, DOI 10.1112/S0010437X04001071. MR2134273 (2006d:14015)

[Bir12] C. Birkar, Singularities on the base of a Fano type fibration, arXiv:1210.2658.

[BB92] A. A. Borisov and L. A. Borisov, Singular toric Fano three-folds (Russian), Mat. Sb. 183 (1992), no. 2, 134-141, DOI 10.1070/SM1993v075n01ABEH003385; English transl., Russian Acad. Sci. Sb. Math. 75 (1993), no. 1, 277-283. MR.1166957 (93i:14034)

[Fuj99] Osamu Fujino, Applications of Kawamata's positivity theorem, Proc. Japan Acad. Ser. A Math. Sci. 75 (1999), no. 6, 75-79. MR1712648(2000f:14089)

[Ful93] William Fulton, Introduction to toric varieties, Annals of Mathematics Studies, vol. 131. The William H. Roever Lectures in Geometry. Princeton University Press, Princeton, NJ, 1993. MR1234037 (94g:14028)

[Hen83] Douglas Hensley, Lattice vertex polytopes with interior lattice points, Pacific J. Math. 105 (1983), no. 1, 183-191. MR688412 (84c:52016)

[Kaw97] Yujiro Kawamata, Subadjunction of log canonical divisors for a subvariety of codimension 2, Birational algebraic geometry (Baltimore, MD, 1996), Contemp. Math., vol. 207, Amer. Math. Soc., Providence, RI, 1997, pp. 79-88, DOI 10.1090/conm/207/02721. MR1462926 (99a:14024)

[Kaw98] Yujiro Kawamata, Subadjunction of log canonical divisors. II, Amer. J. Math. 120 (1998), no. 5, 893-899. MR1646046(2000d:14020)

[KM98] János Kollár and Shigefumi Mori, Birational geometry of algebraic varieties, with the collaboration of C. H. Clemens and A. Corti. Translated from the 1998 Japanese original. Cambridge Tracts in Mathematics, vol. 134, Cambridge University Press, Cambridge, 1998. MR1658959 (2000b:14018)

[KMM87] Yujiro Kawamata, Katsumi Matsuda, and Kenji Matsuki, Introduction to the minimal model problem, Algebraic geometry, Sendai, 1985, Adv. Stud. Pure Math., vol. 10, North-Holland, Amsterdam, 1987, pp. 283-360. MR946243 (89e:14015)

[LZ91] Jeffrey C. Lagarias and Günter M. Ziegler, Bounds for lattice polytopes containing a fixed number of interior points in a sublattice, Canad. J. Math. 43 (1991), no. 5, 1022-1035, DOI 10.4153/CJM-1991-058-4. MR1138580(92k:52032)

[MP08] Shigefumi Mori and Yuri Prokhorov, On $\mathbb{Q}$-conic bundles, Publ. Res. Inst. Math. Sci. 44 (2008), no. 2, 315-369, DOI 10.2977/prims/1210167329. MR.2426350(2009e:14062)

[Oda88] Tadao Oda, Convex bodies and algebraic geometry, An introduction to the theory of toric varieties. Translated from the Japanese. Ergebnisse der Mathematik und ihrer Grenzgebiete (3) [Results in Mathematics and Related Areas (3)], vol. 15, SpringerVerlag, Berlin, 1988. MR.922894 (88m:14038)

Department of Mathematics, University of Georgia, Athens, Georgia 30605

E-mail address: valery@math.uga.edu

Department of Mathematics, 301 Thackeray Hall, University of Pittsburgh, PittsBurgh, Pennsylvania 15260

E-mail address: borisov@pitt.edu 